\title{
Favipiravir May Acts as COVID-19 Main Protease PDB ID 6LU7 Inhibitor: Docking Analysis
}

\author{
Ajeet $^{1 *}$ (D), Babita Aggarwal ${ }^{1}$, Santosh Kumar Verma ${ }^{1}$ \\ 1 Faculty of Pharmaceutical Sciences, Motherhood University, Roorkee, India \\ * Correspondence: ajeet_pharma111@rediffmail.com;
}

Scopus Author ID 55227575600

Received: 18.04.2020; Revised: 23.05.2020; Accepted: 24.05.2020; Published: 27.05.2020

\begin{abstract}
Coronavirus is a well-known threat to the human being in the form of COVID-19. Virus replication may be controlled by inhibition of protease enzyme. Hence, well known 13 antiviral drugs have been observed by docking analysis for understanding the binding pattern of drugs with COVID19 main protease PDB ID: 6LU7 for any possibilities of protease inhibition. For docking analysis PyRxPython Prescription 0.8 was used. This analysis reveals that the essential amino acids involved in binding of antiviral drugs to COVID-19 main protease PDB ID: 6LU7 are Glycine (Gly), Serine (Ser), Cysteine (Cys), Leucine (Leu), Asparagine (Asn), Glutamine (Gln), Glutamic acid (Glu) and Threonine (Thr). After docking analysis, it was observed that Favipiravir maybe act as COVID-19 main protease inhibitor despite being vRNA polymerase inhibitor and may further be used in the treatment of COVID19 infection.
\end{abstract}

Keywords: COVID-19; antiviral; protease inhibition; vRNA polymerase inhibitor.

(C) 2020 by the authors. This article is an open-access article distributed under the terms and conditions of the Creative Commons Attribution (CC BY) license (https://creativecommons.org/licenses/by/4.0/).

\section{Introduction}

Coronavirus is well-known threat peaking its crown again to world in the form of nCoV-19 (COVID-19) during the current time which was initially supposed to have emerged several years ago in different other forms like Middle East Respiratory Syndrome-CoronaVirus (MERS-CoV) and Severe Acute Respiratory Syndrome-corona-virus (SARS-CoV) with the few similar pathological symptoms but with great power of spreading the infection to one another [1]. There is an option out of many for the management of virus is known as inhibition of protease enzyme in virus for the cessation of viral replication. Hence, to contribute a little with some possibilities of inhibition of protease enzyme of COVID-19 virus by existing antiviral drugs; we have screened 13 antiviral drugs which were already approved and tested against their pharmacokinetic, pharmacodynamic and toxicity parameters; for studying their molecular interaction with recently deposited and released crystal structure of COVID-19 main protease (viral protein) with Protein Data Bank (PDB) ID: 6LU7 [2]. The literature revealed that antiviral drugs like Lopinavir, Nelfinavir, etc. which are HIV Protease inhibitors were already used against MERS-CoV and SARS-CoV [3, 4] The antiretroviral protease inhibitors act by binding to the catalytic site of the HIV protease, thereby preventing the cleavage of viral polyprotein precursors into mature, functional proteins that are necessary for viral replication [5]. The drug molecules which were used in this study are Limonin (A naturally occurring tetracyclic triterpenoid derived from the plants of Rutaceae and Meliaceae, known for its antivirus, anti-tumor, anti-inflammatory, analgesic, nerve protection, anti-bacterial, anti-oxidant, liver protection and blood lipid regulation activities. However, they were reported for their 
toxicity also along with poor oral absorption and low bioavailability. It is supposed to have its anti-HIV-1 activity due to inhibition of HIV-1 protease) [6], Pepstatin-A (Naturally occurring aspartyl proteases inhibitor secreted by Streptomyces species.) [7], Ritonavir (Protease inhibitor with HIV-I resistance with good bioavailability; as far as selectivity concerned it is $\geq 500$-fold more selective for HIV aspartic protease as compared to human aspartic protease) [8], Indinavir (HIV protease inhibitor which acts by binding to the HIV protease active site and inhibiting post-translational processing with approx. 95\% of inhibition having maximum drug conc. in plasma in about 0.8 hours) [9], Nelfinavir (It is a selective, nonpeptidic competitive inhibitor for HIV-1 protease and also used as non-nucleoside reverse transcriptase inhibitors. It is also used in the drug-resistant cases, as in the case of Zidovudine. It shows positive effects on immune function with increases in CD4+ cell counts when used in combination with another drug) $[10,11]$, Amprenavir (sulfonamide compound that prevents the formation of HIV-1 virions by inhibiting the viral enzyme protease; rapidly absorbed orally with peak plasma concentration in about 1 to 2 hours. It has the longest plasma elimination half-life time, i.e., 711 hours). CYP 3A4 inhibition is shown by Amprenavir and may interact with inducers, inhibitors or substrates of this system [12], Lopinavir (Also a Protease inhibitor) [13], Atazanavir (It is also potent, and well-tolerated protease inhibitor used to treat HIV in combination with other drugs like ritonavir with effects on lipid parameters) [14-16], Darunavir (It is a non-peptidyl protease inhibitor acts by inhibiting the cleavage of HIV-1 encoded polyproteins. It is used in pediatric patients having HIV infection with different doses) [17-21], Fosamprenavir (It is sulphonamide non-peptide antiretroviral protease inhibitor works by inhibiting cleavage of HIV polyprotein precursor) [22], Temsavir, Raltegravir (It is antiviral, acts by inhibiting integrase enzyme hence blocking the insertion of DNA into host genome) [23-26] and Favipiravir (It is a potent and selective inhibitor of influenza viral RNA polymerase which makes it effective against almost all viruses) [27-30].

To study this interaction between pre-established antiviral drugs and crystal structure of COVID-19 main protease, we have used docking analysis. [31-34]

\section{Materials and Methods}

\subsection{Data, database, and tools.}

For carrying out this study, the National Center for Biotechnology Information's (NCBI) website and Protein Data Bank's (PDB) website were used as biological and chemical data sources. For designing and optimizing the geometry of the derivatives, ChemDraw Ultra 10.0 [31, 35]. Co-crystallized 3D structure of COVID-1 main protease; PDB ID: 6LU7 (viral protein) was downloaded from Protein Data Bank.

\subsection{Docking.}

The docking analysis of 13 antiviral drugs and inhibitor N3 complexes within 3D structure of COVID-19 main protease; PDB ID: 6LU7 was performed by PyRx- Python Prescription 0.8 .

PyRx is Virtual Screening software for Computational Drug Discovery that can be used to screen libraries of compounds against potential drug targets. PyRx enables Medicinal Chemists to run Virtual Screening from any platform and helps users in every step of this process - from data preparation to job submission and analysis of the results. 


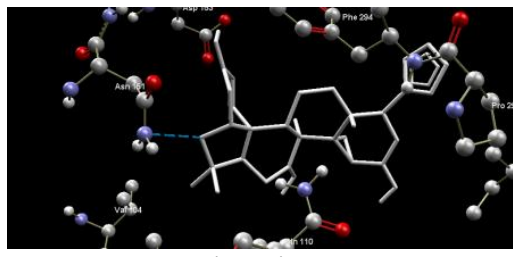

Limonin

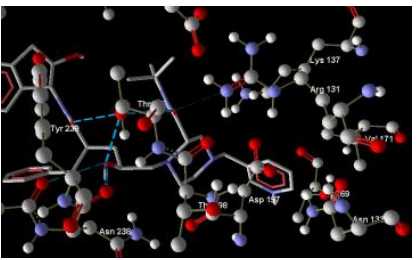

Indinavir

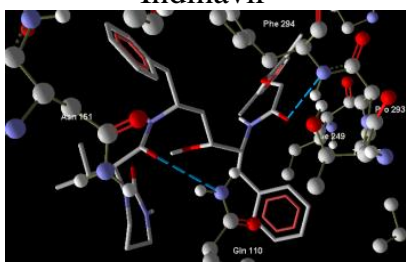

Lopinavir

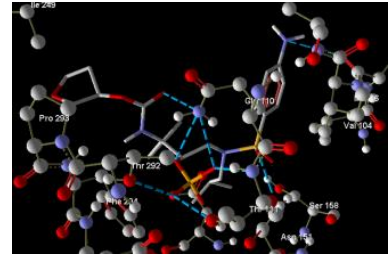

Fosamprenavir

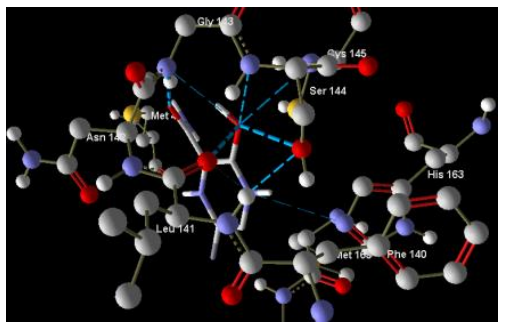

Favipiravir

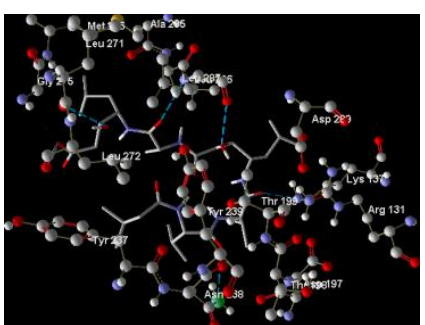

Pepstatin-A

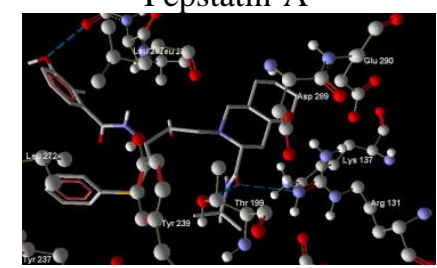

Nelfinavir

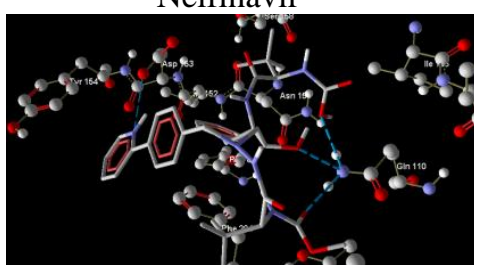

Atazanavir



Temsavir



Ritonavir

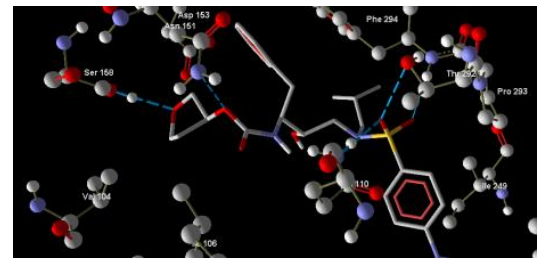

Amprenavir

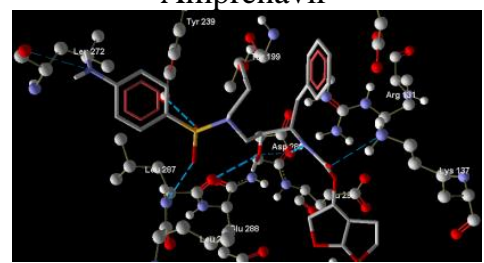

Darunavir

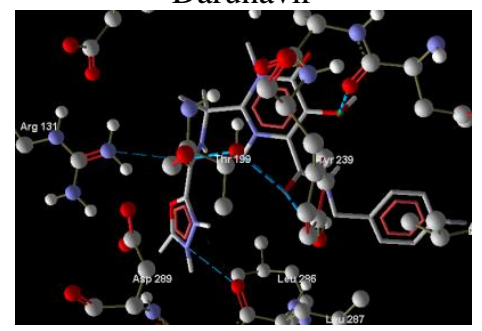

Raltegravir

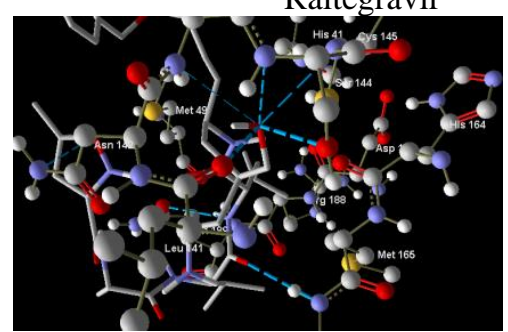

N3(Ref. molecule)

Figure 1. Docked images of antiviral drugs taken and ref. drug complexes in COVID-19 main protease 6LU7

\section{Results and Discussion}

13 antiviral drug molecules were processed for virtual screening via docking studies. All the molecules were analyzed for their binding characteristics such as binding residues, binding affinity energy, number of hydrogen bonds, binding atoms with the type of bonds. All these observed characteristics are given in Table 1, and clicked screenshots of molecular interactions are given in Fig. 1.

Table 1. Docking results of antiviral drugs taken and ref. drug complexes in COVID-19 main protease 6LU7.

\begin{tabular}{|c|c|c|c|c|c|c|c|c|c|}
\hline \multirow[t]{2}{*}{ Ligand } & \multirow{2}{*}{$\begin{array}{l}\text { Affinity } \\
\text { Kcal/mol }\end{array}$} & \multirow{2}{*}{$\begin{array}{c}\text { H- } \\
\text { bonds }\end{array}$} & \multicolumn{3}{|c|}{ H- Binding Ligand } & \multicolumn{4}{|c|}{ H- Binding Receptor } \\
\hline & & & Elem. & At. ID & Type & Residue & Elem. & At.ID & Type \\
\hline \multirow[t]{4}{*}{ Limonin } & -8.2 & 1 & $\mathrm{O}$ & 3 & Acceptor & Asn151 & $\mathrm{N}$ & 1159 & Donor \\
\hline & & & $\mathrm{O}$ & 34 & Acceptor & Thr199 & $\mathrm{O}$ & 1527 & Both \\
\hline & & & $\mathrm{O}$ & 34 & Acceptor & Arg131 & $\mathrm{N}$ & 1016 & Donor \\
\hline & & & $\mathrm{O}$ & 22 & Both & Leu272 & $\mathrm{O}$ & 2102 & Acceptor \\
\hline \multirow[t]{4}{*}{ Pepstatin-A } & -6.1 & 8 & $\mathrm{O}$ & 22 & Both & Gly 275 & $\mathrm{~N}$ & 2124 & Donor \\
\hline & & & $\mathrm{O}$ & 17 & Both & Leu271 & $\mathrm{O}$ & 2094 & Acceptor \\
\hline & & & $\mathrm{O}$ & 26 & Both & Leu287 & $\mathrm{O}$ & 2208 & Acceptor \\
\hline & & & $\mathrm{O}$ & 24 & Acceptor & Leu287 & $\mathrm{N}$ & 2205 & Donor \\
\hline https://biointe & earc & & & & & & & & 6823 \\
\hline
\end{tabular}




\begin{tabular}{|c|c|c|c|c|c|c|c|c|c|}
\hline \multirow[t]{2}{*}{ Ligand } & \multirow{2}{*}{$\begin{array}{c}\text { Affinity } \\
\text { Kcal/mol }\end{array}$} & \multirow{2}{*}{$\begin{array}{c}\text { H- } \\
\text { bonds }\end{array}$} & \multicolumn{3}{|c|}{ H- Binding Ligand } & \multicolumn{4}{|c|}{ H- Binding Receptor } \\
\hline & & & Elem. & At. ID & Type & Residue & Elem. & At.ID & Type \\
\hline \multirow{8}{*}{ Ritonavir } & \multirow{8}{*}{-7.3} & \multirow{8}{*}{6} & $\mathrm{O}$ & 38 & Acceptor & Asn238 & $\mathrm{N}$ & 1852 & Donor \\
\hline & & & $\mathrm{O}$ & 10 & Acceptor & Arg131 & $\mathrm{N}$ & 1016 & Donor \\
\hline & & & $\mathrm{O}$ & 10 & Acceptor & Thr199 & $\mathrm{O}$ & 1527 & Both \\
\hline & & & $\mathrm{O}$ & 27 & Both & Thr199 & $\mathrm{O}$ & 1527 & Both \\
\hline & & & $\mathrm{O}$ & 27 & Both & Tyr237 & $\mathrm{O}$ & 1836 & Acceptor \\
\hline & & & $\mathrm{N}$ & 18 & Donor & Asp197 & $\mathrm{O}$ & 1514 & Acceptor \\
\hline & & & $\mathrm{N}$ & 44 & Donor & Lys236 & & 1827 & Acceptor \\
\hline & & & $\mathrm{O}$ & 36 & Acceptor & Thr199 & $\mathrm{O}$ & 1527 & Both \\
\hline \multirow{5}{*}{ Indinavir } & & & $\mathrm{O}$ & 23 & Acceptor & Thr199 & $\mathrm{O}$ & 1527 & Both \\
\hline & & & $\mathrm{O}$ & 24 & Both & Thr199 & $\mathrm{O}$ & 1527 & Both \\
\hline & -7.9 & 6 & $\mathrm{O}$ & 24 & Both & Asn238 & $\mathrm{O}$ & 1848 & Acceptor \\
\hline & & & $\mathrm{O}$ & 24 & Both & Tyr237 & $\mathrm{O}$ & 1836 & Acceptor \\
\hline & & & $\mathrm{O}$ & 23 & Acceptor & Arg131 & $\mathrm{N}$ & 1016 & Donor \\
\hline & & & $\mathrm{O}$ & 37 & Acceptor & $\operatorname{Arg} 131$ & $\mathrm{~N}$ & 1016 & Donor \\
\hline Nelfinavir & -7.5 & 3 & $\mathrm{O}$ & 37 & Acceptor & Thr199 & $\mathrm{O}$ & 1527 & Both \\
\hline & & & $\mathrm{O}$ & 11 & Both & Ala285 & $\mathrm{O}$ & 2195 & Acceptor \\
\hline & & & $\mathrm{O}$ & 8 & Acceptor & Thr292 & $\mathrm{O}$ & 2255 & Both \\
\hline & & & $\mathrm{O}$ & 8 & Acceptor & Gln110 & $\mathrm{N}$ & 861 & Donor \\
\hline Amprenavir & -7.6 & 5 & $\mathrm{O}$ & 9 & Acceptor & Phe294 & $\mathrm{N}$ & 2264 & Donor \\
\hline & & & $\mathrm{O}$ & 32 & Acceptor & Asn151 & $\mathrm{N}$ & 1159 & Donor \\
\hline & & & $\mathrm{O}$ & 36 & Acceptor & Ser158 & $\mathrm{O}$ & 1214 & Both \\
\hline & & 2 & $\mathrm{O}$ & 4 & Acceptor & Gln110 & $\mathrm{N}$ & 861 & Donor \\
\hline Lopinavir & -8.1 & 2 & $\mathrm{O}$ & 38 & Acceptor & Phe294 & $\mathrm{N}$ & 2264 & Donor \\
\hline & & & $\mathrm{O}$ & 48 & Acceptor & Gln110 & $\mathrm{N}$ & 861 & Donor \\
\hline Atazanavir & & 4 & $\mathrm{O}$ & 26 & Both & Gln110 & $\mathrm{N}$ & 861 & Donor \\
\hline Atazanavır & -6.9 & 4 & $\mathrm{O}$ & 11 & Acceptor & Gln110 & $\mathrm{N}$ & 861 & Donor \\
\hline & & & $\mathrm{N}$ & 36 & Donor & Asp153 & $\mathrm{O}$ & 1174 & Acceptor \\
\hline & & & $\mathrm{N}$ & 29 & Donor & Asp289 & $\mathrm{O}$ & 2228 & Acceptor \\
\hline & & & $\mathrm{O}$ & 19 & Both & Asp289 & $\mathrm{O}$ & 2228 & Acceptor \\
\hline & & & $\mathrm{O}$ & 19 & Both & Leu287 & $\mathrm{O}$ & 2208 & Acceptor \\
\hline & & & $\mathrm{O}$ & 31 & Acceptor & Lys137 & $\mathrm{N}$ & 1066 & Donor \\
\hline Darunavir & -1.1 & 8 & $\mathrm{O}$ & 8 & Acceptor & Leu287 & $\mathrm{N}$ & 2205 & Donor \\
\hline & & & $\mathrm{O}$ & 9 & Acceptor & Tyr239 & $\mathrm{O}$ & 1864 & Both \\
\hline & & & $\mathrm{N}$ & 16 & Donor & Leu272 & $\mathrm{O}$ & 2102 & Acceptor \\
\hline & & & $\mathrm{O}$ & 37 & Acceptor & Lys5 & $\mathrm{N}$ & 40 & Donor \\
\hline & & & $\mathrm{O}$ & 34 & Acceptor & Gln110 & $\mathrm{N}$ & 861 & Donor \\
\hline & & & $\mathrm{O}$ & 19 & Acceptor & Gln110 & $\mathrm{N}$ & 861 & Donor \\
\hline & & & $\mathrm{O}$ & 22 & Acceptor & Gln110 & $\mathrm{N}$ & 861 & Donor \\
\hline & & & $\mathrm{O}$ & 22 & Acceptor & Thr111 & $\mathrm{N}$ & 862 & Donor \\
\hline Fosamprenavir & -7.3 & 9 & $\mathrm{O}$ & 8 & Acceptor & Asn151 & $\mathrm{N}$ & 1159 & Donor \\
\hline & & & $\mathrm{O}$ & 9 & Acceptor & Asn151 & $\mathrm{N}$ & 1159 & Donor \\
\hline & & & $\mathrm{O}$ & 23 & Acceptor & Thr111 & $\mathrm{O}$ & 867 & Both \\
\hline & & & $\mathrm{O}$ & 23 & Acceptor & Thr292 & $\mathrm{O}$ & 2255 & Both \\
\hline & & & $\mathrm{N}$ & 16 & Donor & Arg105 & $\mathrm{O}$ & 817 & Acceptor \\
\hline & & & $\mathrm{O}$ & 12 & Acceptor & Thr199 & $\mathrm{O}$ & 1527 & Both \\
\hline Temsavir & -7.7 & 3 & $\mathrm{O}$ & 37 & Acceptor & Thr199 & $\mathrm{O}$ & 1527 & Both \\
\hline & & & $\mathrm{N}$ & 31 & Donor & Leu271 & $\mathrm{O}$ & 2094 & Acceptor \\
\hline & & & $\mathrm{O}$ & 14 & Acceptor & Thr199 & $\mathrm{O}$ & 1527 & Both \\
\hline & & & $\mathrm{O}$ & 25 & Acceptor & Thr199 & $\mathrm{O}$ & 1527 & Both \\
\hline & & & $\mathrm{O}$ & 25 & Acceptor & Tyr239 & $\mathrm{O}$ & 1864 & Both \\
\hline Raltegravir & -8.7 & 7 & $\mathrm{O}$ & 14 & Acceptor & Arg131 & $\mathrm{N}$ & 1016 & Donor \\
\hline & & & $\mathrm{N}$ & 17 & Donor & Leu287 & $\mathrm{O}$ & 2208 & Acceptor \\
\hline & & & $\mathrm{N}$ & 16 & Donor & Leu287 & $\mathrm{O}$ & 2208 & Acceptor \\
\hline & & & $\mathrm{O}$ & 36 & Both & Tyr237 & $\mathrm{O}$ & 1836 & Acceptor \\
\hline & & & $\mathrm{O}$ & 14 & Both & Cys145 & $\mathrm{N}$ & 2231 & Donor \\
\hline & & & $\mathrm{O}$ & 14 & Both & Ser144 & $\mathrm{N}$ & 2220 & Donor \\
\hline & & & $\mathrm{O}$ & 14 & Both & Gly143 & $\mathrm{N}$ & 2213 & Donor \\
\hline & & & $\mathrm{O}$ & 14 & Both & Leu141 & $\mathrm{O}$ & 2183 & Acceptor \\
\hline Favipiravir & -5.3 & 9 & $\mathrm{O}$ & 14 & Both & Ser144 & $\mathrm{O}$ & 2225 & Both \\
\hline & & & $\mathrm{O}$ & 13 & Acceptor & Gly143 & $\mathrm{N}$ & 2213 & Donor \\
\hline & & & $\mathrm{N}$ & 2 & Donor & Leu141 & $\mathrm{O}$ & 2183 & Acceptor \\
\hline & & & $\mathrm{N}$ & 2 & Donor & Ser144 & $\mathrm{O}$ & 2225 & Both \\
\hline & & & $\mathrm{N}$ & 2 & Donor & His163 & $\mathrm{N}$ & 2497 & Acceptor \\
\hline & & & $\mathrm{O}$ & 52 & Both & Gly143 & $\mathrm{N}$ & 2213 & Donor \\
\hline & & & $\mathrm{O}$ & 52 & Both & Ser144 & $\mathrm{N}$ & 2220 & Donor \\
\hline N3 & -6.5 & 9 & $\mathrm{O}$ & 52 & Both & Cys 145 & $\mathrm{~N}$ & 2231 & Donor \\
\hline & & & $\mathrm{O}$ & 52 & Both & Ser144 & $\mathrm{O}$ & 2225 & Both \\
\hline & & & $\mathrm{O}$ & 52 & Both & Leu141 & $\mathrm{O}$ & 2183 & Acceptor \\
\hline s://bioi & & & & & & & & & 6824 \\
\hline
\end{tabular}




\begin{tabular}{cccccccccc}
\hline \multirow{2}{*}{ Ligand } & Affinity & H- & \multicolumn{4}{c}{ H- Binding Ligand } & \multicolumn{3}{c}{ H- Binding Receptor } \\
\cline { 3 - 9 } & Kcal/mol & bonds & Elem. & At. ID & Type & Residue & Elem. & At.ID & Type \\
\cline { 3 - 9 } & & & $\mathrm{O}$ & 20 & Acceptor & Asn142 & $\mathrm{N}$ & 2206 & Donor \\
& & $\mathrm{N}$ & 1 & Donor & Gln189 & $\mathrm{O}$ & 2880 & Acceptor \\
& & & $\mathrm{O}$ & 3 & Acceptor & Glu166 & $\mathrm{N}$ & 2539 & Donor \\
& & & $\mathrm{O}$ & 34 & Both & Thr26 & $\mathrm{O}$ & 363 & Acceptor \\
\cline { 3 - 11 } & & & & & & & & &
\end{tabular}

Initial analysis showed that highest number of hydrogen bond (9) with COVID-19 main protease; PDB ID: 6LU7 was formed by Fosamprenavir (Binding affinity energy: -7.3 $\mathrm{Kcal} / \mathrm{mol}$ ) and Favipiravir (Binding affinity energy: $-5.3 \mathrm{Kcal} / \mathrm{mol}$ ), but lowest binding affinity energy $-8.7 \mathrm{Kcal} / \mathrm{mol}$ (Required as lowest as possible) was observed with Raltegravir (Number of Hydrogen bond: 7). But, initial observations are not sufficient in order to make finding any decisive clue for opting for the best one.

Hence, further Residual (Binding Amino acids) analysis has been performed. This comparative analysis was performed with the residues of inhibitor N3 complexes within the 3D structure of COVID-19 main protease; PDB ID: 6LU7. This analysis is given in Table 2. On analyzing Table 2, it was observed that Amino acids or residues which plays a key role in the binding of reference molecule N3 are Glycine (Gly), Serine (Ser), Cysteine (Cys), Leucine (Leu), Asparagine (Asn), Glutamine (Gln), Glutamic acid (Glu) and Threonine (Thr).

Antiviral drugs Pepstatin-A (associated amino acids are- Gly, Leu, Asn, and Thr), Amprenavir (associated amino acids are- Ser, Asn, Gln, and Thr) and Favipiravir (associated amino acids are- Gly, Ser, Cys, and Leu) were found to be very close to the binding pocket of protein 6LU7.

These 3 screened drug molecules have been further analyzed for exact residual matching with reference drug N3. See Table 3. This shows that only Favipiravir (associated amino acids are- Gly143, Ser144, Cys145, and Leu141) is the drug molecule out of 13 studied antiviral drugs found somewhat exactly in the same pocket as that of reference drug molecule N3 complexes within the 3D structure of COVID-19 main protease 6LU7. Although Favipiravir is a viral RNA polymerase inhibitor, it shows better binding towards the COVID-19 main protease also.

Table 2. Residual analysis of docked antiviral drugs.

\begin{tabular}{|c|c|c|c|c|c|c|c|c|c|c|}
\hline \multirow[t]{2}{*}{ Type } & \multirow{2}{*}{$\begin{array}{l}\begin{array}{c}\text { Ligands } \\
\text { (Antiviral } \\
\text { Drugs) }\end{array} \\
\text { N3 (Ref. } \\
\text { molecule) }\end{array}$} & \multicolumn{8}{|c|}{ Essential Residues } & \multirow{2}{*}{$\begin{array}{l}\text { No. of Residues } \\
\text { matching with ref. } \\
\text { Molecule }\end{array}$} \\
\hline & & Gly & Ser & Cys & Leu & Asn & Gln & Glu & Thr & \\
\hline \multirow{12}{*}{$\begin{array}{l}\text { Protease } \\
\text { Inhibitor }\end{array}$} & Limonin & - & - & - & - & Yes & - & - & - & 1 \\
\hline & Pepstatin-A & Yes & - & - & Yes & Yes & - & - & Yes & 4 \\
\hline & Ritonavir & - & - & - & - & - & - & - & Yes & 1 \\
\hline & Indinavir & - & - & - & - & Yes & - & - & Yes & 2 \\
\hline & Nelfinavir & - & - & - & - & - & - & - & Yes & 1 \\
\hline & Amprenavir & - & Yes & - & - & Yes & Yes & - & Yes & 4 \\
\hline & Lopinavir & - & - & - & - & - & Yes & - & - & 1 \\
\hline & Atazanavir & - & - & - & - & - & Yes & - & - & 1 \\
\hline & Darunavir & - & - & - & Yes & - & - & - & - & 1 \\
\hline & Fosamprenavir & - & - & - & - & Yes & Yes & - & Yes & 3 \\
\hline & Temsavir & - & - & - & Yes & - & - & - & Yes & 2 \\
\hline & Raltegravir & - & & - & Yes & - & - & - & Yes & 2 \\
\hline vRNA & Favipiravir & & & & & & & & & \\
\hline $\begin{array}{l}\text { Polymerase } \\
\text { Inhibitor }\end{array}$ & & Yes & Yes & Yes & Yes & - & - & - & - & 4 \\
\hline
\end{tabular}




\section{Conclusions}

Favipiravir, which is a vRNA polymerase inhibitor, has shown its possibilities of having inhibition of COVID-19 main protease enzyme also; this enzyme is responsible for replication of the virus in the living body. To reach this conclusion, we have completed a journey of docking analysis of 13 well known antiviral drugs with COVID-19 main protease PDB ID: 6LU7. Docking was performed with the help of PyRx- Python Prescription 0.8, and the results were observed and analyzed, which initially screened 3 (Pepstatin-A, Amprenavir, and Favipiravir) out of 13 antiviral drugs having possibilities of protease inhibition of virus. The further residual analysis showed that Favipiravir might have probabilities of protease inhibition for the cessation of viral replication as amino acids of the binding pocket (Gly143, Ser144, Cys145, and Leu141 out of 8 amino acids) for Favipiravir were similar to reference molecule N3 complexes within the 3D structure of COVID-19 main protease 6LU7. These results primarily showed the possibilities only, although clinical studies are still required to ascertain the findings.

Table 3. Exact Residual analysis of 3 screened antiviral drugs.

\begin{tabular}{lccccccccc}
\hline \multicolumn{1}{c}{ Ligands } & \multicolumn{7}{c}{ Exact Match of Essential Residues } & $\begin{array}{c}\text { No. of Residues } \\
\text { matching with ref. } \\
\text { Molecule }\end{array}$ \\
\hline $\begin{array}{l}\text { N3 (Ref. } \\
\text { molecule) }\end{array}$ & Gly143 & Ser144 & Cys145 & Leu141 & Asn142 & Gln189 & Glu166 & Thr26 & - \\
\hline Pepstatin-A & - & - & - & - & - & - & - & - & - \\
Amprenavir & - & - & - & - & - & - & - & - & 4 \\
Favipiravir & Yes & Yes & Yes & Yes & - & - & - & - & \\
\hline
\end{tabular}

\section{Funding}

This research received no external funding.

\section{Acknowledgments}

Authors are thankful to CBBE (Computational Biology for Biochemical Experiments, https://www.cbbe-rnd.com/), In-silico Drug Design and Development Services, India, for cross analyzing the data.

\section{Conflicts of Interest}

The authors declare no conflict of interest.

\section{References}

1. Al-Hazmi, A. Challenges presented by MERS corona virus, and SARS corona virus to global health. Saudi Journal of Biological Sciences 2016, 23, 507-511,https://doi.org/10.1016/j.sjbs.2016.02.019.

2. RCSB PDB - 6LU7: The crystal structure of COVID-19 main protease in complex with an inhibitor N3. https://www.rcsb.org/structure/6lu7.(accessed on13 April2020). https://doi.org/10.2210/pdb6LU7/pdb.

3. Momattin, H.; Al-Ali, A.Y.; Al-Tawfiq, J.A. A Systematic Review of therapeutic agents for the treatment of the Middle East Respiratory Syndrome Coronavirus (MERS-CoV). Travel Medicine and Infectious Disease 2019, 30, 9-18, https://doi.org/10.1016/j.tmaid.2019.06.012.

4. Totura, A.L.; Bavari, S. Broad-spectrum coronavirus antiviral drug discovery. Expert Opinion on Drug Discovery 2019, 14, 397-412, https://doi.org/10.1080/17460441.2019.1581171.

5. Protease Inhibitors (HIV).In: LiverTox: Clinical and Research Information on Drug-Induced Liver Injury [Internet]. Bethesda (MD): National Institute of Diabetes and Digestive and Kidney Diseases. 2012-.2017

6. Fan, S.; Zhang, C.; Luo, T.; Wang, J.; Tang, Y.; Chen, Z.; Yu, L. Limonin: A Review of Its Pharmacology, Toxicity, and Pharmacokinetics. Molecules 2019, 24,https://doi.org/10.3390/molecules24203679. 
7. Mothes, E.; Shoeman, R.L.; Traub, P. Pepstatin A: Polymerization of an oligopeptide. Micron 1994, 25, 189217, https://doi.org/10.1016/0968-4328(94)90042-6.

8. Lea, A.P.; Faulds, D.; Ritonavir. Drugs1996, 52, 541-546,https://doi.org/10.2165/00003495-19965204000007.

9. Pollak, E.B.; Parmar, M. Indinavir.In: StatPearls [Internet]. Treasure Island (FL): StatPearls Publishing. 2020 Jan-.2020 Jan 24.

10. Perry, C.M.; Frampton, J.E.; McCormack, P.L.; Siddiqui, M.A.A.; Cvetković, R.S. Nelfinavir. Drugs 2005, 65, 2209-2244,https://doi.org/10.2165/00003495-200565150-00015.

11. Perry, C.M.; Benfield, P. Nelfinavir. Drugs 1997, 54, 81-87, https://doi.org/10.2165/00003495-19975401000007.

12. Noble, S.; Goa, K.L. Amprenavir: a review of its clinical potential in patients with HIV infection. Drugs 2000, 60, 1383-1410, https://doi.org/10.2165/00003495-200060060-00012.

13. Yao, T.-T.; Qian, J.-D.; Zhu, W.Y.; Wang, Y.; Wang, G.-Q. A systematic review of lopinavir therapy for SARS coronavirus and MERS coronavirus-A possible reference for coronavirus disease-19 treatment option. Journal of Medical Virology 2020, 92, 556-563, https://doi.org/10.1002/jmv.25729.

14. Hara, M.; Suganuma, A.; Yanagisawa, N.; Imamura, A.; Hishima, T.; Ando, M. Atazanavir nephrotoxicity. Clinical Kidney Journal 2015, 8, 137-142, https://doi.org/10.1093/ckj/sfv015.

15. Cure, S.; Bianic, F.; Espinas, C.; Hardy, H.; Rosenblatt, L.; Juday, T. Systematic Literature Review and Meta-Analysis of Renal Function in Human Immunodeficiency Virus (HIV)-Infected Patients Treated with Atazanavir (ATV)-Based Regimens. PLOS ONE e0124666,https://doi.org/10.1371/journal.pone.0124666.

16. Menshawy, A.; Ismail, A.; Abushouk, A.I.; Ahmed, H.; Menshawy, E.; Elmaraezy, A.; Gadelkarim, M.; Abdel-Maboud, M.; Attia, A.; Negida, A. Efficacy and safety of atazanavir/ritonavir-based antiretroviral therapy for HIV-1 infected subjects: a systematic review and meta-analysis. Archives of Virology 2017, 162, 2181-2190, https://doi.org/10.1007/s00705-017-3346-9.

17. Keating, G.M. Darunavir: A Review in Pediatric HIV-1 Infection. Pediatric Drugs 2015, 17, 411-421, https://doi.org/10.1007/s40272-015-0146-0.

18. Khoo, S.; Peytavin, G.; Burger, D.; Hill, A.; Brown, K.; Moecklinghoff, C.; La Porte, C.; Hadacek, M.B. Pharmacokinetics and Safety of Darunavir/Ritonavir in HIV-Infected Pregnant Women. AIDS Rev 2017, 19, 16-23.

19. Colombier, M.-A.; Molina, J.-M. Doravirine: a review. Current Opinion in HIV and AIDS 2018, 13, 308314,https://doi.org/10.1097/COH.0000000000000471.

20. Spagnuolo, V.; Castagna, A.; Lazzarin, A. Darunavir for the treatment of HIV infection. Expert Opinion on Pharmacotherapy 2018, 19, 1149-1163, https://doi.org/10.1080/14656566.2018.1484901.

21. Deeks, E.D. Darunavir/Cobicistat/Emtricitabine/Tenofovir Alafenamide: A Review in HIV-1 Infection. Drugs 2018, 78, 1013-1024, https://doi.org/10.1007/s40265-018-0934-2.

22. Fosamprenavir. In:LiverTox: Clinical and Research Information on Drug-Induced Liver Injury, National Institute of Diabetes and Digestive and Kidney Diseases: Bethesda (MD), 2012.

23. Gutierrez-Valencia, A.; Chacón-Mora, N.; Ruiz-Valderas, R.; Ben-Marzouk-Hidalgo, O.J.; Torres-Cornejo, A.; Viciana, P.; Lopez-Cortes, L.F. Does Once-Daily Raltegravir Have Any Role in the Antiretroviral Treatment? Medicine 2015, 94.https://doi.org/10.1097/MD.0000000000001743.

24. Deeks, E.D. Raltegravir Once-Daily Tablet: A Review in HIV-1 Infection. Drugs 2017, 77, 17891795,https://doi.org/10.1007/s40265-017-0827-9.

25. Maliakkal, A.; Walmsley, S.; Tseng, A. Critical Review: Review of the Efficacy, Safety, and Pharmacokinetics of Raltegravir in Pregnancy. JAIDS Journal of Acquired Immune Deficiency Syndromes 2016, 72. 153-161. https://doi.org/10.1097/QAI.0000000000000932.

26. de Miguel, R.; Montejano, R.; Stella-Ascariz, N.; Arribas, J.R. A safety evaluation of raltegravir for the treatment of HIV. Expert Opinion on Drug Safety 2018, 17, 217223,https://doi.org/10.1080/14740338.2018.1411903.

27. Furuta, Y.; Komeno, T.; Nakamura, T. Favipiravir (T-705), a broad spectrum inhibitor of viral RNA polymerase. Proceedings of the Japan Academy, Series B 2017, 93, 449463,https://doi.org/10.2183/pjab.93.027

28. Furuta, Y.; Gowen, B.B.; Takahashi, K.; Shiraki, K.; Smee, D.F.; Barnard, D.L. Favipiravir (T-705), a novel viral RNA polymerase inhibitor. Antiviral Research 2013, 100, 446-454, https://doi.org/10.1016/j.antiviral.2013.09.015. 
29. Du, Y.X.; Chen, X.P. Favipiravir: Pharmacokinetics and Concerns About Clinical Trials for 2019-nCoV infection. Clinical Pharmacology \& Therapeutics 2020,https://doi.org/10.1002/cpt.1844.

30. Shiraki, K.; Daikoku, T. Favipiravir, an anti-influenza drug against life-threatening RNA virus infections. Pharmacology \& Therapeutics 2020, 209, https://doi.org/10.1016/j.pharmthera.2020.107512.

31. Ajeet, A.; Kumar, A.; Mishra, A. Design, Synthesis and Pharmacological Evaluation of Sulfonamide Derivatives Screened Against Maximal Electroshock Seizure Test. Molecular Biology 2018, 7, 206. http://doi.org/10.4172/2168-9547.1000206.

32. Ajeet; Kumar, A.; Mishra, A.K. Design, molecular docking, synthesis, characterization, biological activity evaluation (against MES model), in-silico biological activity spectrum (PASS analysis), toxicological and predicted oral rat LD50 studies of novel sulphonamide derivatives. Frontiers in Biology 2018, 13, 425451,https://doi.org/10.1007/s11515-018-1512-4.

33. Ajeet. In silico designing and characterization of Amiloride derivatives as ion channel modulator. Medicinal Chemistry Research 2013, 22, 1004-1010,http://doi.org/10.1007/s00044-012-0096-9.

34. Ajeet; Verma, M.; Rani, S.; Kumar, A. Antitarget Interaction, Acute Toxicity and Protein Binding Studies of Quinazolinedione Sulphonamides as GABA1 Antagonists. Indian J Pharm Sci 2016, 78, 4853,http://doi.org/10.4103/0250-474x.180249.

35. Mills, N. ChemDraw Ultra 10.0. J Am Chem Soc 2006, 128, 1364913650,https://doi.org/10.1021/ja0697875. 